\title{
An analysis of AVO inversion for postcritical offsets in HTI media
}

\author{
Lyubov Skopintseva $^{1}$ and Tariq Alkhalifah ${ }^{2}$
}

\begin{abstract}
Azimuthal variations of wavefield characteristics, such as traveltime or reflection amplitude, play an important role in the identification of fractured media. A transversely isotropic medium with a horizontal symmetry axis (HTI medium) is the simplest azimuthally anisotropic model typically used to describe one set of vertical fractures. There exist many techniques in industry to recover anisotropic parameters based on moveout equations and linearized reflection coefficients using such a model. However, most of the methods have limitations in defining properties of the fractures due to linearizations and physical approximations used in their development. Thus, azimuthal analysis of traveltimes based on normal moveout ellipses recovers a maximum of three medium parameters instead of the required five. Linearizations made in plane-wave reflection coefficients (PWRCs) limit the amplitude-versus-offset (AVO) analysis to small incident angles and weak-contrast interfaces. Inversion based on azimuthal AVO for small offsets encounters nonuni-
\end{abstract}

queness in the resolving power of the anisotropy parameters. Extending the AVO analysis and inversion to and beyond the critical reflection angle increases the amount of information recovered from the medium. However, well-accepted PWRCs are not valid in the vicinity of the critical angle and beyond it, due to frequency and spherical wave effects. Recently derived spherical and effective reflection coefficient (ERC) methods overcome this problem. We extended the ERCs approach to HTI media to analyze the potential of near- and postcritical reflections in azimuthal AVO analysis. From the sensitivity analysis, we found that ERCs are sensitive to different sets of parameters prior to and beyond the critical angle, which is useful in enhancing our resolution of the anisotropy parameters. Additionally, the resolution of the parameters depends on a sufficient azimuthal coverage in the acquisition setup. The most stable AVO results for the azimuthal acquisition setup with minimum number of lines (three) are achieved when the azimuthal angle between lines is greater than $45^{\circ}$.

\section{INTRODUCTION}

The role of anisotropy has dramatically increased over the past two decades due to advances in acquisition setups, data quality, data processing, and parameter estimation. It has been demonstrated that including anisotropy in the data analysis considerably reduces uncertainty in interpretation (Tsvankin et al., 2010). Fracture identification, direction, and density estimation have become more attainable with the use of multiazimuth acquisition setup and multiazimuth data analysis. However, although we see that fractured reservoirs often adhere closely to the orthorhombic symmetry (Grechka et al., 2006), azimuthal analysis based on HTI symmetry is widely exploited for a vertical set of fractures. The HTI is the simplest azimuthally anisotropic model (Rüger, 2001).
One of the most widely exploited approaches in fracture identification is the azimuthal analysis of reflection traveltimes based on the concept of the normal moveout (NMO) ellipse (Grechka et al., 1999). Although P-wave azimuthal moveout analysis is practically effective in predicting the fracture direction (Lynn et al., 1999; Tod et al., 2007), the NMO ellipse constrains only three combinations of the medium parameters, leading naturally to a three-parameter inversion (Al-Dajani and Alkhalifah, 2000). Azimuthally dependent $\mathrm{P}$-wave traveltime inversion recovers vertical P-wave velocity, anisotropy parameter $\delta^{(V)}$ (or anellipticity parameter $\eta$; Alkhalifah and Tsvankin, 1995), and the symmetry axis direction. The NMO ellipse, however, is not enough to fully characterize five parameters of an HTI model.

\footnotetext{
Manuscript received by the Editor 1 August 2011; revised manuscript received 27 September 2012; published online 12 April 2013.

${ }^{1}$ Norwegian University of Science and Technology, Department of Petroleum Engineering and Applied Geophysics, Trondheim, Norway. E-mail: lyus@ statoil.com.

${ }^{2}$ King Abdullah University of Science and Technology, Thuwal, Saudi Arabia. E-mail: tariq.alkhalifah@kaust.edu.sa.

(c) 2013 Society of Exploration Geophysicists. All rights reserved.
} 
Reflection coefficients contain valuable information about the local medium properties on both sides of an interface. Therefore, analysis of amplitude variations with incidence angle or offset is often used in reservoir characterization (Mallick and Frazer, 1991; Mallick et al., 1998; Avseth et al., 2001). Generally, amplitude-versusoffset (AVO) analysis has higher vertical resolution than traveltime methods. The AVO inversion approaches used in industry are based on the linearizations of plane-wave reflection coefficients (PWRCs), made under the assumption of weak contrast interfaces (Ostrander, 1984). As a consequence of linearizations, application of such AVO approaches is limited to small offsets, where a reasonably good match with real data is achieved. The application of azimuthally dependent approximations (Rüger, 2001) in azimuthal AVO inversion, however, is hindered by nonuniqueness in parameter estimation. Practically, azimuthal variations of AVO response are exploited in the recovery of a fracture azimuth with a $90^{\circ}$ uncertainty (Hall and Kendall, 2003). Despite this ambiguity, azimuthal AVO analysis showed promise in many cases (Gray et al., 2002; Hall and Kendall, 2003; Xu and Tsvankin, 2007).

Nonconventional reservoirs such as stiff-carbonate reservoirs, heavy oil traps, salt domes, and basalts represent a challenge in the oil industry today. These reservoirs are characterized by high contrasts in media parameters across the interface. This results in reflections associated with the critical angle at relatively small offsets, where conventional AVO analysis is not valid anymore. The importance of near- and postcritical reflections is highlighted by several researchers (Downton and Ursenbach, 2006; Alhussain, 2007; Skopintseva et al., 2011), who show that using such reflections improves inversion results. Based on PWRC analysis, Hall and Kendall (2003) and Landrø and Tsvankin (2007) notice that the critical angle is sensitive to the fracture orientation.

However, PWRCs do not describe near- and postcritical reflections generated by a point source, which are functions of the wavefront radius and frequency (Červený, 1961; Alhussain, 2007). Recently developed spherical and effective reflection coefficients (ERCs) for isotropic/isotropic interfaces (Ayzenberg et al., 2007; Ursenbach et al., 2007) provide better insights of the reflection behavior observed around and beyond the critical angle. Extension of ERCs to isotropic/VTI interfaces (Ayzenberg et al., 2009) shows their sensitivity to anisotropy parameters.

In this paper, we investigate the potential of using postcritical reflections in azimuthal AVO analysis and inversion. For this purpose, we extend ERCs to an isotropic/HTI interface and compare their azimuthal dependence prior to and beyond the critical angle. We show that the amplitude maximum observed beyond the critical angle can be used in anisotropy parameter estimation. We provide sensitivity analysis of reflection coefficient sensitivity to the underburden parameters prior to and beyond the critical angle for multiazimuth acquisition coverage with a minimal number of survey lines (three). Results indicate that pre- and postcritical domains are sensitive to different sets of parameters. In addition, we provide analysis for the optimal acquisition setup for better stability of the inversion.

\section{PHASE VELOCITY SURFACE VERSUS CRITICAL ANGLE SURFACE}

Consider a two-layer model with a plane interface, in which the upper half-space is isotropic and the lower half-space represents an HTI medium. The P-wave velocity of the isotropic half-space is lower than the P-wave phase velocity of the HTI medium for any azimuthal direction. For simplicity, assume that the S-wave phase velocity in the underburden is constant and lower than the $\mathrm{P}$-wave velocity in the overburden. When $\mathrm{P}$-wave propagating in the upper half-space hits the isotropic/HTI interface at the critical angle, part of the energy reflects from the interface, part of it converts to the transmitted SV-wave, and the rest of energy starts propagating along the interface and generates a PPP head wave (Figure 1). The velocity of the head wave corresponds to the horizontal P-wave phase velocity of the underburden, and it is the function of angle $\varphi$ between the incidence plane and the HTI symmetry axis. Therefore, the critical angle $\theta_{\mathrm{cr}}$ is also a function of azimuth $\varphi$ due to the modified Snell's law (Landrø and Tsvankin, 2007):

$$
\sin \theta_{\mathrm{cr}}(\varphi)=\frac{V_{\mathrm{P} 1}}{V_{\mathrm{P} 2}^{h}(\varphi)},
$$

where $V_{\mathrm{P} 1}$ is the $\mathrm{P}$-wave velocity in the overburden and $V_{\mathrm{P} 2}^{h}(\varphi)$ is the azimuthally dependent horizontal phase $\mathrm{P}$-wave velocity in the underburden. The plot in Figure 1 defines the critical angles for two orthogonal incidence planes. Plane $\|$ is located along the symmetry axis of an HTI medium, and plane $\perp$ coincides with the isotropic plane of an HTI medium. $V_{\mathrm{P} 2}^{h}(0)$ corresponds to the phase velocity along the symmetry axis, and $V_{\mathrm{P} 2}^{h}(90)=V_{\mathrm{P} 2}$ is the phase $\mathrm{P}$-wave velocity in the isotropic plane $\perp$. The horizontal velocity in plane $\|$ is less than the horizontal velocity in plane $\perp$. Consequently, the critical angle in the plane along the symmetry axis is larger than that for the isotropic plane. The reciprocal proportionality of the sine of the critical angle to the horizontal phase velocity (equation 1) shows that the azimuthal dependence of the critical angle might bring an additional information about the underburden.

Azimuthal dependency of the horizontal P-wave phase velocity surface for HTI media is equivalent to the P-wave phase velocity surface for VTI media in the vertical plane because an HTI medium is equivalent to a VTI medium rotated by $90^{\circ}$ in the vertical plane. According to well-known acoustic approximations (S-wave velocities are assumed to be zero) for the phase velocity in VTI media (Tsvankin, 2005), the azimuthally dependent horizontal phase velocity for an HTI medium has the following form:

$$
\begin{aligned}
& V_{\mathrm{P} 2}^{h 2}(\varphi)=V_{\mathrm{P} 2}^{2}\left[\frac{1}{2}+\epsilon^{(V)} \cos ^{2} \varphi\right. \\
& \left.+\frac{1}{2} \sqrt{1+4 \cos ^{2} \varphi\left[\epsilon^{(V)} \cos 2 \varphi+2 \delta^{(V)} \sin ^{2} \varphi\right]+4 \epsilon^{(V) 2} \cos ^{4} \varphi}\right],
\end{aligned}
$$

where $\epsilon^{(V)}$ and $\delta^{(V)}$ are anisotropy parameters in HTI notation (Rüger, 2001). Note that the horizontal P-wave velocity does not depend on the third anisotropy parameter $\gamma^{(V)}$, which results in nonsensitivity of the critical angle to this parameter.

Substituting equation 1 into 2 and exploiting the three azimuthal directions corresponding to $\varphi=0^{\circ}, 45^{\circ}$, and $90^{\circ}$ yields the following equations for anisotropy parameters in terms of their critical angles:

$$
\begin{aligned}
\epsilon^{(V)} & =\frac{\sin ^{2} \theta_{\mathrm{cr}}(90)-\sin ^{2} \theta_{\mathrm{cr}}(0)}{2 \sin ^{2} \theta_{\mathrm{cr}}(0)} \\
\delta^{(V)} & =\frac{\sin ^{2} \theta_{\mathrm{cr}}(90)\left[\sin ^{2} \theta_{\mathrm{cr}}(45)-2 \sin ^{2} \theta_{\mathrm{cr}}(0)\right]\left[\sin ^{2} \theta_{\mathrm{cr}}(45)-2 \sin ^{2} \theta_{\mathrm{cr}}(90)\right]}{2 \sin ^{2} \theta_{\mathrm{cr}}(0) \sin ^{2} \theta_{\mathrm{cr}}(45)}-\frac{1}{2} .
\end{aligned}
$$

To obtain anisotropy parameter $\epsilon^{(V)}$, we need information about the critical angles in the incidence planes along and across the symmetry 
axis of HTI media. Anisotropy parameter $\delta^{(V)}$ requires additional knowledge about the critical angle for an azimuth of $45^{\circ}$. Equation 3 implies that the symmetry axis direction is known. The symmetry axis direction can be estimated from the azimuthal distribution of the critical angle under two assumptions: parameter $\epsilon$ has the stronger impact on $V_{\mathrm{P} 2}^{h}(0)$ than $\delta$ and the horizontal P-wave velocity along the symmetry axis direction $V_{\mathrm{P} 2}^{h}(0)$ is smaller than horizontal $\mathrm{P}$-wave velocity in the isotropic plane $V_{\mathrm{P} 2}^{h}(90)$. The second assumption implies that symmetry axis direction corresponds to the largest value of the critical angle.

\section{EFFECTIVE REFLECTION COEFFICIENT FOR THE ISOTROPIC/HTI INTERFACE}

Here, we extend the ERCs to the isotropic/HTI case. We choose the incidence plane coinciding with the $\left(x_{1}, x_{3}\right)$ plane of the global coordinate system $\left(x_{1}, x_{2}, x_{3}\right)$ and forming an angle $\varphi$ with the symmetry axis of an HTI medium. A point source exciting a spherical $\mathrm{P}$-wave is located in the upper half-space. The ERC represents a ratio of the reflected spherical wave to the incident spherical wave (Ayzenberg et al., 2007). For the isotropic/HTI interface at the point of a receiver, it has the form similar to ERC for isotropic/VTI interface (Ayzenberg et al., 2009):

$\chi_{P P}[\mathbf{x}, \omega, \mathrm{g}]=\frac{u_{P P \text { norm }}^{*}(\mathbf{x}, \mathrm{g}) \cos \theta(\mathbf{x})+u_{P P \tan }^{*}(\mathbf{x}, \mathrm{g}) \sin \theta(\mathbf{x})}{\left[\frac{i}{k_{P} r_{P P}^{*}(\mathbf{x})}-\frac{1}{k_{P}^{2} r_{P P}^{* 2}(\mathbf{x})}\right] e^{i k_{P} r_{P P}^{*}(\mathbf{x})}}$,

where $i$ is the imaginary part, matrix $\mathbf{x}=\left(\mathrm{x}_{1}, \mathrm{x}_{2}, \ldots, \mathrm{x}_{N}\right)$ consists of $N$ source-receiver offsets in 3D space $\mathrm{x}_{N}=\left(x_{1 N}, x_{2 N}, x_{3 N}\right) ; \omega$ is the angular frequency; $\mathrm{g}=\left(V_{\mathrm{P} 1}, V_{\mathrm{S} 1}, \rho_{1}, V_{\mathrm{P} 2}, V_{\mathrm{S} 2}, \epsilon^{(V)}, \gamma^{(V)}, \delta^{(V)}\right.$, $\left.\varphi, \rho_{2}\right)$ is the vector of model parameters; $V_{\mathrm{P} 1}, V_{\mathrm{S} 1}$ are the $\mathrm{P}$ and $\mathrm{S}$-wave velocities of the isotropic half-space; $V_{\mathrm{P} 2}$ and $V_{\mathrm{S} 2}$ are the P-and S-wave velocities of the HTI model in the isotropic plane; $\rho_{1}, \rho_{2}$ are the densities of the upper and lower half-spaces, respectively; $\epsilon^{(V)}, \gamma^{(V)}, \delta^{(V)}$ are the anisotropy parameters in HTI notation (Rüger, 2001); $k_{P}=\frac{\omega}{V_{\mathrm{Pl}}}$ is the wavenumber in the overbur$\operatorname{den} ; \theta(\mathbf{x})$ is the incidence/reflection angle; and $r_{P P}^{*}(\mathbf{x})$ is the apparent radius of the wavefront at the receiver. A general form of the radius $r_{P P}^{*}(\mathbf{x})$ is introduced by Skopintseva et al. (2012). For a plane interface, $r_{P P}^{*}(\mathbf{x})$ is the distance between the source and the receiver along the ray (Skopintseva et al., 2011). The dimensionless normal and tangential components of the displacement vector are represented by $u_{P P \text { norm }}^{*}(\mathbf{x})$ and $u_{P P \tan }^{*}(\mathbf{x})$, which have the following form:

$$
\begin{gathered}
u_{P P \text { norm }}^{*}(\mathbf{x}, \mathrm{g})=-\int_{0}^{+\infty} R_{P P}(\zeta, \mathrm{g}) e^{i \alpha(\mathbf{x}) \sqrt{1-\zeta^{2}}} J_{0}[\beta(\mathbf{x}) \zeta] \zeta d \zeta, \\
u_{P P \tan }^{*}(\mathbf{x}, \mathrm{g})=-\int_{0}^{+\infty} R_{P P}(\zeta, \mathrm{g}) \frac{i e^{i \alpha(\mathbf{x}) \sqrt{1-\zeta^{2}}}}{\sqrt{1-\zeta^{2}}} J_{1}[\beta(\mathbf{x}) \zeta] \zeta^{2} d \zeta,
\end{gathered}
$$

where $R_{P P}(\zeta, \mathrm{g})$ is an exact PWRC for the isotropic/HTI interface derived by Schoenberg and Protazio (1992), $\alpha(\mathbf{x})=$ $k_{P} r_{P P}^{*}(\mathbf{x}) \cos \theta(\mathbf{x}), \beta(\mathbf{x})=k_{P} r_{P P}^{*}(\mathbf{x}) \sin \theta(\mathbf{x}), \zeta$ is the horizontal component of the unit $\mathrm{P}$-wave ray vector in the overburden, and $J_{0}$ and $J_{1}$ are the Bessel functions of the zeroth and first order.

Equations 4 and 5 show that reflection coefficient at the receiver does not depend on one reflection point as it is assumed in plane wave theory. The reflection process for spherical waves includes the contribution of all point of the interface. The largest contribution, however, comes from reflection points of the interface included into the Fresnel zone (Ayzenberg et al., 2007). Equations 4-5 can be easily transferred from the offset domain to the angle domain. We exploit the offset domain mostly for convenient representation of the reflection coefficient at the postcritical domain.

Figure 2 shows the amplitude of the ERC normalized by the reflection amplitude at the first receiver $\mathrm{x}_{1}(A(\mathbf{x}, \varphi)=$ $\left.\chi(\mathbf{x}, \varphi) / \chi\left(\mathrm{x}_{1}, \varphi\right)\right)$. It is compared with amplitudes of the normalized PWRC $A_{R}(\mathbf{x}, \varphi)=R_{P P}(\mathbf{x}, \varphi) / R_{P P}\left(\mathrm{x}_{1}, \varphi\right)$ and reflection coefficient obtained from reflectivity modeling using the technique described by Skopintseva et al. (2011). Reflection coefficients are calculated for the model water/HTI provided by Alhussain (2007), where $V_{\mathrm{P} 1}=1.484 \mathrm{~km} / \mathrm{s}, \quad V_{\mathrm{S} 1}=0 \mathrm{~km} / \mathrm{s}, \quad \rho_{1}=1 \mathrm{~g} / \mathrm{cm}^{3}, \quad V_{\mathrm{P} 2}=$ $2.709 \mathrm{~km} / \mathrm{s}, \quad V_{\mathrm{S} 2}=1.382 \mathrm{~km} / \mathrm{s}, \quad \rho_{2}=1.2 \mathrm{~g} / \mathrm{cm}^{3}, \quad \epsilon^{(V)}=$ $-0.0019, \delta^{(V)}=-0.0069, \gamma^{(V)}=-0.0439, \varphi=30^{\circ}$, the frequency is $218 \mathrm{~Hz}$, and the interface depth is $240 \mathrm{~m}$. The ERC and PWRC curves coincide at precritical angles, whereas they are different around and beyond the critical angle. The ERC has a gradual amplitude increase with the angle and reaches its maximum beyond the critical angle, whereas the PWRC has an abrupt amplitude increase with the maximum at the critical angle. Additionally, the ERC has oscillations in the postcritical domain, whereas these are absent for the PWRC. Effects observed for the ERC around and beyond the critical angle occur due to the influence of the dimensionless argument $k_{P} r_{P P}^{*}(\mathbf{x})$ in equations $4-5$. The perfect match of the ERC with the reflection coefficient extracted from the synthetic data confirms the soundness of the observed phenomena described by the ERCs. This fact allows exploiting equations for ERC to study the influence of different model parameters on the amplitude behavior for pre-, near-, and postcritical offsets without computing incredible amount of synthetic seismic gathers. Moreover, these equations can be easily exploited in the long-offset AVO inversion.

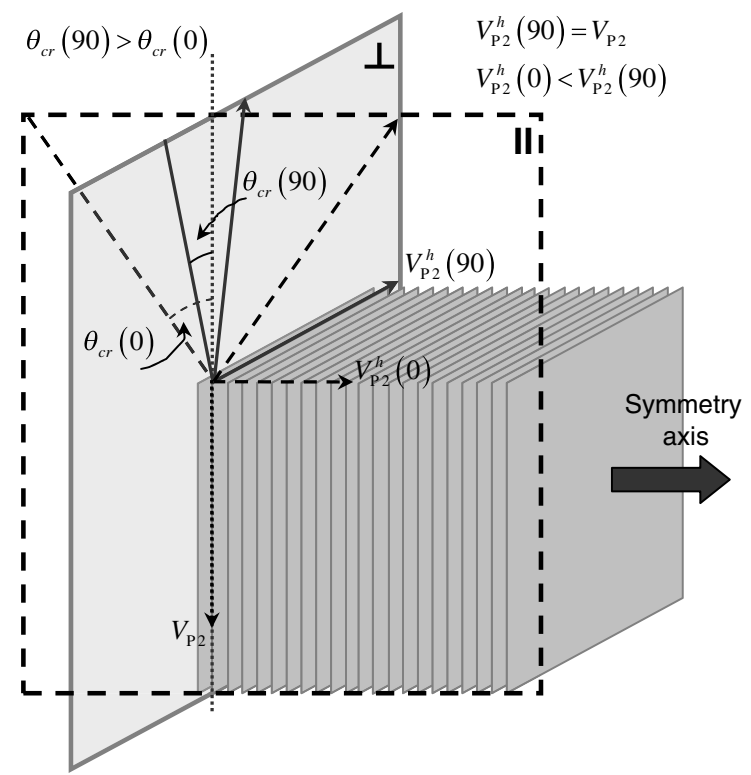

Figure 1. Schematic plot depicting the model setting considered here. The ray curves correspond to a reflection at the critical angle from the horizontal isotropic/HTI interface. 
The argument $k_{P} r_{P P}^{*}(\mathbf{x})$ represents the effect of the wavefront curvature and frequency on the reflection amplitude. Its value controls the amplitude maximum shift and amplitude oscillation in postcritical domain associated with the interference between reflected and head waves (Figure 3). Taking into account that the argument $k_{P} r_{P P}^{*}(\mathbf{x})$ is the composite of the wavefront curvature and the frequency, the effect of this argument on the reflection coefficient has a dual physical insight. Thus, the infinite value of $k_{P} r_{P P}^{*}(\mathbf{x})$ can be associated with either the infinite frequency or with the zero-wavefront curvature, which represents the assumptions of plane wave theory. Therefore, the ERC asymptotically tends to the PWRC with the argument $k_{P} r_{P P}^{*}(\mathbf{x})$ approaching infinity. For plane interfaces, the argument $k_{P} r_{P P}^{*}(\mathbf{x})$ can be represented as the effect of traveltime and frequency on the reflection amplitude because $k_{P} r_{P P}^{*}(\mathbf{x})=\omega t_{P P}(\mathbf{x})$, where $t_{P P}$ is the traveltime. In this situation,



Figure 2. Comparison between the normalized ERC, the PWRC, and the reflection response obtained from reflectivity modeling. Reflection coefficients are calculated for the model with $V_{\mathrm{P} 1}=1.484 \mathrm{~km} / \mathrm{s}, \quad V_{\mathrm{S} 1}=0 \mathrm{~km} / \mathrm{s}$, and $\rho_{1}=1 \mathrm{~g} / \mathrm{cm}^{3}$ in the overburden and $V_{\mathrm{P} 2}=2.709 \mathrm{~km} / \mathrm{s}, \quad V_{\mathrm{S} 2}=1.382 \mathrm{~km} / \mathrm{s}, \quad \rho_{2}=$ $1.2 \mathrm{~g} / \mathrm{cm}^{3}, \epsilon^{(V)}=-0.0019, \delta^{(V)}=-0.0069$, and $\gamma^{(V)}=-0.0439$ in the underburden. The azimuthal angle of the acquisition line relative to the symmetry axis is $\varphi=30^{\circ}$. The frequency of the signal is $218 \mathrm{~Hz}$, and the interface depth is $240 \mathrm{~m}$.

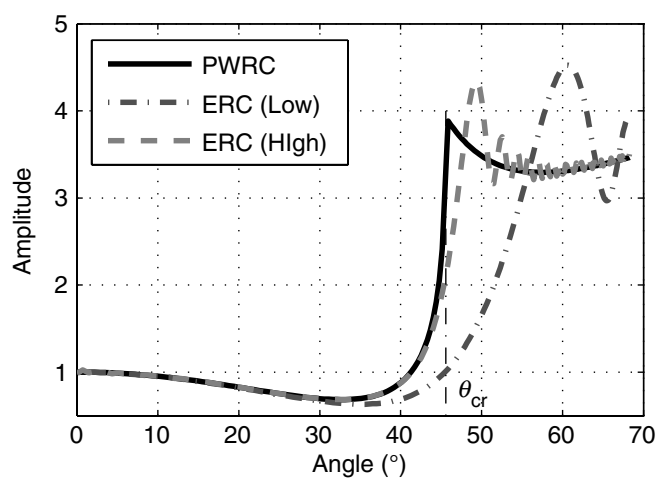

Figure 3. Amplitude of normalized ERCs in the isotropic plane $\varphi=90^{\circ}$ for $k_{P} r_{P}^{*}(0)=402$ (high) and $k_{P} r_{P}^{*}(0)=25$ (low). The argument $k_{P} r_{P}^{*}$ represents the product of the raypath length and wavenumber for the isotropic/HTI plane interface. The normalized PWRC is given for comparison. The model parameters are as follows: $V_{\mathrm{P} 1}=2 \mathrm{~km} / \mathrm{s}, \quad V_{\mathrm{S} 1}=1.1 \mathrm{~km} / \mathrm{s}, \rho_{1}=1.8 \mathrm{~g} / \mathrm{cm}^{3}, V_{\mathrm{P} 2}=$ $2.8 \mathrm{~km} / \mathrm{s}, V_{\mathrm{S} 2}=1.6 \mathrm{~km} / \mathrm{s}, \rho_{2}=2.1 \mathrm{~g} / \mathrm{cm}^{3}, \epsilon^{(V)}=-0.1, \delta^{(V)}=$ -0.05 , and $\gamma^{(V)}=-0.1$. the ERC tends to PWRC with the frequency or traveltime approaching infinity.

Figures 4 and 5 show maps of azimuthal distributions of the normalized reflection coefficients for different anisotropy parameters $\epsilon^{(V)}, \delta^{(V)}, \gamma^{(V)}$ and a frequency of $32 \mathrm{~Hz}$. We used a model in which the isotropic half-space with parameters $V_{\mathrm{P} 1}=2 \mathrm{~km} / \mathrm{s}, V_{\mathrm{S} 1}=$ $1.1 \mathrm{~km} / \mathrm{s}$, and $\rho_{1}=1.8 \mathrm{~g} / \mathrm{cm}^{3}$ overlays the HTI half-space with background parameters $V_{\mathrm{P} 2}=2.8 \mathrm{~km} / \mathrm{s}, V_{\mathrm{S} 2}=1.6 \mathrm{~km} / \mathrm{s}$, and $\rho_{2}=2.1 \mathrm{~g} / \mathrm{cm}^{3}$ at a depth of $1 \mathrm{~km}$. The change in velocity across the interface is representative for fractured reservoirs with strong contrast interfaces. The isotropic overburden is chosen to avoid anisotropy effects on traveltime. This ensures that the argument $k_{P} r_{P P}^{*}(\mathbf{x})=\omega t_{P P}$ is anisotropy independent. Therefore, we analyze the effect of anisotropy on reflection amplitudes only. The argument $k_{P} r_{P P}^{*}(\mathbf{x})$ changes within the ranges of 201-284 (Figure 4) and 201-635 (Figure 5) and introduces the degree of deviation of the ERC from PWRC in the postcritical domain. For each row, we change only one anisotropy parameter in the underburden while all others are set to zero. The left column represents a weak anisotropy effect, whereas the right column corresponds to strong anisotropy. The color indicates the amplitude strength. The offset increases in the radial direction from the center. The circle at $1 \mathrm{~km}$ (or, equivalently, $k_{P} r_{P P}^{*}(\mathbf{x})=224$ ) corresponds to an incident/reflection angle of $30^{\circ}$ (if anisotropy parameters are zeros), which is normally a limit for conventional AVO studies. The circle at $2 \mathrm{~km}$ (or, equivalently, $k_{P} r_{P P}^{*}(\mathbf{x})=284$ ) corresponds to the critical angle, if anisotropy parameters are zeros. The azimuthal angle $\varphi$ changes from $0^{\circ}$ to $360^{\circ}$. The offset range in Figure 4 corresponds to the precritical domain, whereas Figure 5 covers pre-, near-, and postcritical offsets.

In the case of the isotropic medium, we expect a uniform amplitude response with azimuth for any offset range (circles). Anisotropy produces deviation from such an isotropic pattern. Figure 4 shows that the parameter $\epsilon^{(V)}$ does not cause much azimuthal deviation in the amplitude within the circle of $1 \mathrm{~km}$. Its effect appears at larger offsets, specifically when the incident angle reaches critical angles. The influence of the parameter $\delta^{(V)}$ is slightly stronger within the circle of $1 \mathrm{~km}$, but larger offsets experience greater influence of this parameter. The largest effect on the azimuthal distribution of amplitudes in Figure 4 is caused by the anisotropy parameter $\gamma^{(V)}$. The amplitude strength deviates from an isotropic pattern at all offsets within the range $0-2 \mathrm{~km}$.

Figure 5 shows well-defined amplitude maximum contour associated with the postcritical domain. This contour, however, does not coincide with the offset corresponding to the critical angle due to the influence of the finite argument $k_{P} r_{P P}^{*}(\mathbf{x})$. The shape of these contours is somewhat proportional to the azimuthal dependence of the critical angle position. Sensitivity of the postcritical domain to the anisotropy parameters $\epsilon^{(V)}$ and $\delta^{(V)}$ is clearly observed, whereas sensitivity to the parameter $\gamma^{(V)}$ is not obvious. Changes in the anisotropy parameter $\epsilon^{(V)}$ control the amplitude maximum deviation from the isotropic circle in the symmetry axis direction $\left(\varphi=0^{\circ}\right)$. Parameter $\delta^{(V)}$ influences the amplitude maximum deviation in the oblique direction $\left(\varphi=45^{\circ}\right)$, as well as amplitude strength for $\varphi=0^{\circ}$ and $\varphi=90^{\circ}$. These observations are consistent with equations 3 , where $\epsilon^{(V)}$ is a function of the critical angles along and across the symmetry axis and $\delta^{(V)}$ is a function of critical angles for three azimuths $\left(\varphi=0^{\circ}, 45^{\circ}, 90^{\circ}\right)$. Parameter $\gamma^{(V)}$ is independent of the critical angle, which follows from equation 2. 
The largest anisotropic effect is observed due to the $\epsilon$ influence, whereas $\delta$ represents the second order effect on the postcritical domain. Therefore, the assumption that the largest critical angle corresponds to the symmetry axis direction sounds reasonable.

\section{CRITICAL OFFSET VERSUS AMPLITUDE MAXIMUM OFFSET}

Figure 2 shows that the critical angle (offset) cannot be clearly defined because it is not associated with special amplitude features of the ERC. However, offsets corresponding to maximum amplitude contours as shown in Figure 5 can be exploited for anisotropy parameter detection. Assume that the deviations of the amplitude maximum position from the critical offset are controlled only by parameter $k_{P} r_{P P}^{*}(\mathbf{x})$ and are weakly dependent on the anisotropy parameters. Then the azimuthal dependence of the critical offset


Figure 4. Maps of the normalized azimuthal ERCs prior to the critical offset $\left(2 \mathrm{~km}\right.$ in the isotropic plane $\left.\varphi=90^{\circ}\right)$ for different anisotropy parameters. The radial direction corresponds to the source-receiver offset from 0 to $2 \mathrm{~km}$, and the angular direction corresponds to the angle between the survey line and the symmetry axis direction from $0^{\circ}$ to $360^{\circ}$. Each row represents changes in one of the anisotropy parameters, while the others are set to zero. is proportional to the azimuthal dependence of the amplitude maximum offset $\mathrm{x}_{m}$ :

$$
\mathrm{x}_{\mathrm{cr}}(\varphi)=n \mathrm{x}_{m}(\varphi)
$$

where $n$ is an azimuthally independent proportionality coefficient, $\mathrm{x}_{\mathrm{cr}}(\varphi)$ is an azimuthally dependent critical offset, and $\mathrm{x}_{m}(\varphi)$ is the azimuthally dependent amplitude maximum offset.

Applying the assumption of equation 6 and the relation

$$
\sin \theta_{\mathrm{cr}}^{2}=\frac{\mathrm{x}_{\mathrm{cr}}^{2}}{h^{2}+\mathrm{x}_{\mathrm{cr}}^{2}},
$$

where $h$ is the interface depth, we rewrite equations 3 in terms of the amplitude maximum offset $\mathrm{x}_{m}$ :
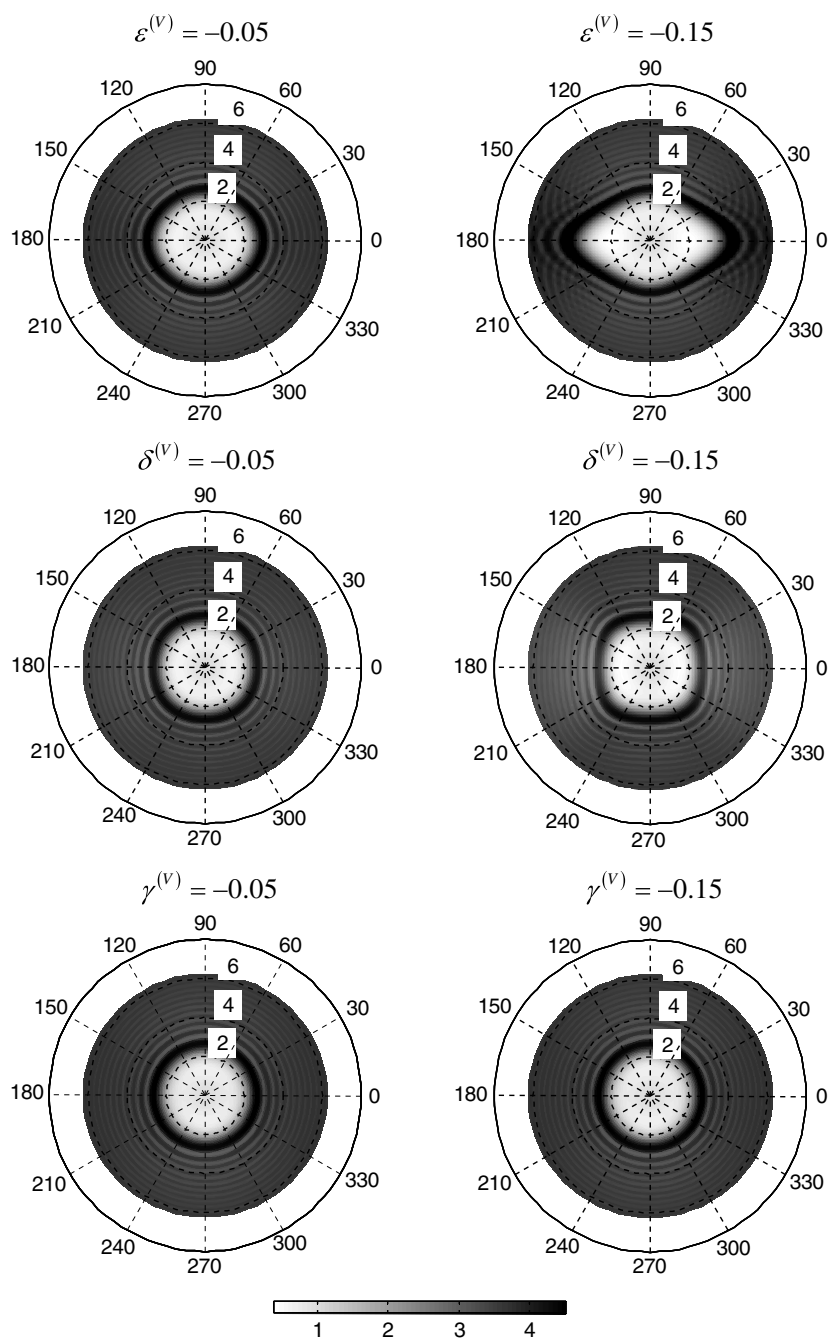

Figure 5. Maps of the normalized azimuthal ERCs prior to and beyond the critical offset for different anisotropy parameters. The radial direction corresponds to the source-receiver offset from 0 to $6 \mathrm{~km}$, and the angular direction corresponds to the angle between the survey line and the symmetry axis direction from $0^{\circ}$ to $360^{\circ}$. Each row represents changes in one of the anisotropy parameters, while the others are set to zero. 


$$
\begin{aligned}
& \epsilon^{(V)}=\cos ^{2} \theta_{\mathrm{cr}}(90)\left[\frac{\mathrm{x}_{m}^{2}(90)-\mathrm{x}_{m}^{2}(0)}{2 \mathrm{x}_{m}^{2}(0)}\right], \\
& \delta^{(V)}=-M_{1} \cos ^{2} \theta_{\mathrm{cr}}(90)+M_{2} \cos ^{2} \theta_{\mathrm{cr}}(90) \cos 2 \theta_{c r}(90),
\end{aligned}
$$

where

$$
\begin{aligned}
& M_{1}=1-\frac{\mathrm{x}_{m}^{2}(90)}{2 \mathrm{x}_{m}^{2}(45)}-\frac{\mathrm{x}_{m}^{4}(90)}{\mathrm{x}_{m}^{4}(45)}+\frac{\mathrm{x}_{m}^{4}(90)}{2 \mathrm{x}_{m}^{2}(0) \mathrm{x}_{m}^{2}(45)}, \\
& M_{2}=\frac{1}{2}-\frac{3 \mathrm{x}_{m}^{2}(90)}{2 \mathrm{x}_{m}^{2}(45)}+\frac{\mathrm{x}_{m}^{4}(90)}{\mathrm{x}_{m}^{4}(45)}+\frac{\mathrm{x}_{m}^{2}(90)}{2 \mathrm{x}_{m}^{2}(0)}-\frac{\mathrm{x}_{m}^{4}(90)}{2 \mathrm{x}_{m}^{2}(0) \mathrm{x}_{m}^{2}(45)} .
\end{aligned}
$$

The azimuthally independent proportionality coefficient $n$ in equations 8-9 is vanished. Although equations 8-9 are functions of the amplitude maximum offsets, the critical angle in the isotropic plane is still needed. This information can be retrieved from an AVO inversion in the isotropic plane (Skopintseva et al., 2011).

Relative errors in anisotropy parameter estimates obtained from the different types of data are shown in Figure 6. The three data sets are exploited for estimations: azimuthal dependency of the horizontal phase velocity $V_{\mathrm{P} 2}^{h}(\varphi)$ given by equation 1 , azimuthal dependency of the critical angle $\theta_{\mathrm{cr}}(\varphi)$ estimated from PWRCs, and azimuthal dependency of the maximum amplitude offset $\mathrm{x}_{m}(\varphi)$ obtained from ERCs. The most accurate estimates are obtained for the anisotropy parameter $\epsilon^{(V)}$. The error level is within $1 \%$ for any type of data, and it is the smallest for horizontal P-wave velocity. Higher
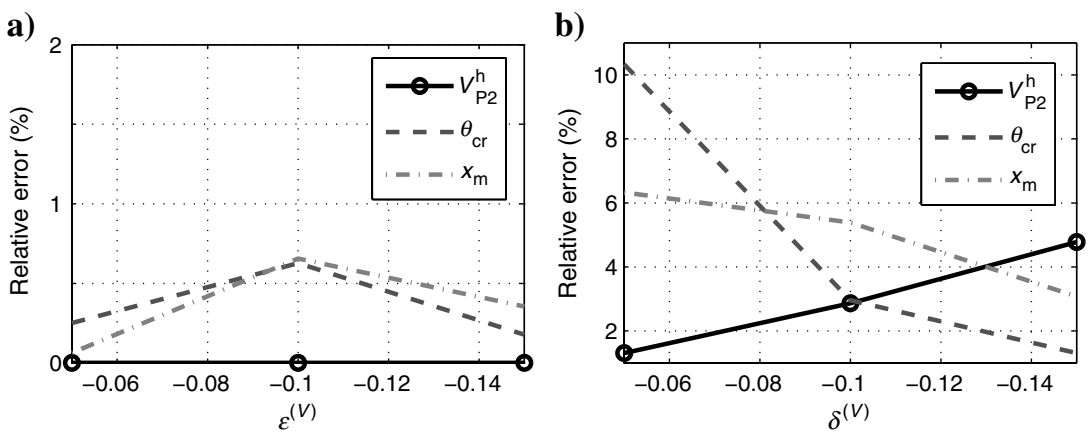

Figure 6. Relative errors in the anisotropy parameter $\epsilon^{(V)}$ (a) and $\delta^{(V)}$. (b) Estimates obtained from velocity, critical angle, and offsets corresponding to the maximum amplitudes.

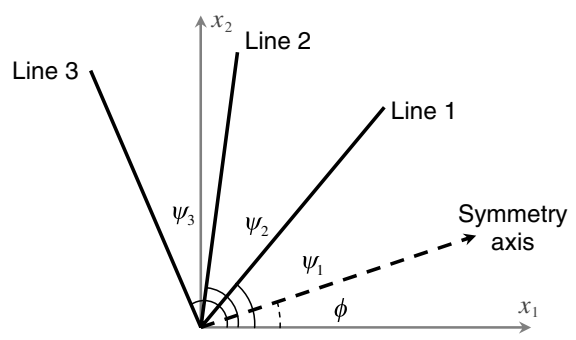

$$
\mathbf{d}=\left(\frac{V_{\mathrm{P} 2} \partial_{V_{\mathrm{P} 2}} A}{A}, \frac{V_{\mathrm{S} 2} \partial_{V_{\mathrm{S} 2}} A}{A}, \frac{\rho_{2} \partial_{\rho_{2}} A}{A}, \frac{\partial_{\epsilon^{(V)}} A}{A}, \frac{\partial_{\delta^{(V)}} A}{A}, \frac{\partial_{\gamma^{(V)}} A}{A}, \frac{2 \pi \partial_{\phi} A}{A}\right)
$$

are submatrices of derivatives of the normalized reflection coefficient $A(\mathbf{x}, \theta-\phi)$ with respect to the medium parameters for a particular survey line and $T$ indicates the transpose of a matrix. The derivatives with respect to velocity and density are normalized to allow for direct comparison with the dimensionless anisotropic parameters.

As a result, the resolution matrix $\mathbf{M}=\mathbf{J}^{T} \mathbf{J}$ provides information on the linear dependency of the parameters and the strength of their resolution. Perfect resolution is given by the identity matrix, which
Figure 7. A plan view of three 2D survey lines over a horizontal HTI layer with the arbitrary symmetry axis direction from the chosen global coordinate system. inaccuracies for $\theta_{\mathrm{cr}}(\varphi)$ and $\mathrm{x}_{m}(\varphi)$ are explained by PWRC and ERC estimates increases up to $10 \%$. The reason for such inaccuracies is the acoustic approximation of phase velocity used in anisotropy parameter estimates. The approximation is least accurate at an of data. In general, the amplitude maximum offset $\mathrm{x}_{m}$ provides a similar level of errors as the critical angle and thus has the potential to be used for anisotropy analysis. Nevertheless, no explicit link maximum has been found yet. It is clear that the argument hip $(\mathbf{x})$ plays the key role. The development of explicit relationcritical domain.

The results of the previous section give the potential for anisotropy parameter analysis only in the case of full azimuthal coverage. from the azimuthal dependence of the amplitude maximum position as seen in Figure 5. However, quite often, data are available only for cation is not obvious.

To understand the potential for using postcritical reflections in quisition coverage with a minimal number of lines, given by angles with coordinate axis $x_{1}$. For simplicity, we assume that the azimuthal separation between the survey lines $\Delta \psi$ is equal and, thus, satisfies parameters of the isotropic overburden are known, and we investigate the sensitivity of the normalized reflection coefficients for an isoparameters of the HTI half-space. To conduct the sensitivity analysis, we exploit techniques described by Al-Dajani and Alkhalifah (2000) and uild up the following Jacobian matrix:

$$
\mathbf{J}^{T}=\left(\mathbf{d}\left(\psi_{1}, \mathbf{x}\right) \mathbf{d}\left(\psi_{2}, \mathbf{x}\right) \mathbf{d}\left(\psi_{3}, \mathbf{x}\right)\right),
$$

where 
indicates that all parameters are resolvable within the linear limit and do not have trade-offs between each other. However, the resolution matrix only allows for a linearized analysis of the sensitivities, valid around a point in the model space. Depending on the level of nonlinearity, this analysis can also be representative of general behavior.

Figure 8 represents the resolution matrix $\mathbf{M}$ for all underburden model parameters for precritical (top row) and postcritical (bottom row) offsets for the following acquisition setups: $\psi_{2}=30^{\circ}$ and $\Delta \psi=60^{\circ}$. Medium parameters in the isotropic upper layer are $V_{\mathrm{P} 1}=2.0 \mathrm{~km} / \mathrm{s}, \quad V_{\mathrm{S} 1}=1.1 \mathrm{~km} / \mathrm{s}$, and $\rho_{1}=$ $1.8 \mathrm{~g} / \mathrm{cm}^{3}$. The parameters of the underburden are chosen to be $V_{\mathrm{P} 2}=2.8 \mathrm{~km} / \mathrm{s}, \quad V_{\mathrm{S} 2}=$ $1.6 \mathrm{~km} / \mathrm{s}, \rho_{2}=2.1 \mathrm{~g} / \mathrm{cm}^{3}, \gamma^{(V)}=-0.1$, and $\phi=0^{\circ}$. Each column corresponds to different combinations of anisotropy parameters $\epsilon^{(V)}$ and $\delta^{(V)}$. Higher diagonal values indicate higher sensitivity of the normalized reflection coefficients to the particular parameter, which consequently results in higher resolution of this parameter in the inversion. The nonzero offdiagonal element reveals a trade-off between parameters related to this element.

Generally, we observe that the sensitivity of medium parameters is model dependent, but there are common trends for different combinations of $\epsilon^{(V)}$ and $\delta^{(V)}$. Normalized ERCs are sensitive to different sets of parameters at pre- and postcritical offsets. Exploiting only the pre critical domain results in resolving $\mathrm{P}$ - and $\mathrm{S}$ wave velocities with reasonable trade-off, and anisotropy parameters $\delta^{(V)}$ and $\gamma^{(V)}$ with some trade-off between them, as well. The sensitivity of the reflection coefficients to the symmetry axis direction in the precritical domain is highly dependent on the strength of anisotropy. In the postcritical domain, the vertical $\mathrm{P}$-wave velocity remains highly resolved with less trade-off with the shear wave velocity, whereas the resolution of $\delta^{(V)}$ and $\gamma^{(V)}$ decreases. Instead, the resolution of density, anisotropy parameter $\epsilon^{(V)}$, and the symmetry axis direction increases considerably. We observe trade-offs in the postcritical domain between $V_{\mathrm{P} 2}$ and $\rho_{2}, \phi$ and $\epsilon^{(V)}$, and $\phi$ and $V_{\mathrm{P} 2}$. However, it is important to note that the symmetry axis resolution is high with a minor trade-off with other parameters, which implies the importance of the postcritical reflection coefficients in resolving the symmetry axis direction.

Figure 9 gives an idea of how azimuth $\psi_{2}$ and separation angle $\Delta \psi$ between the survey lines affect the resolution of some parameters in the precritical (top row) and the postcritical (bottom row) domains. Only diagonal values of the matrix $\mathbf{M}=\mathbf{J}^{T} \mathbf{J}$ corresponding to $V_{\mathrm{P} 2}, \epsilon^{(V)}, \delta^{(V)}$, and $\phi$ are exploited for this purpose. The calculations are made for a model in which the parameters of the isotropic half-space are the same as in Figure 8, and parameters of the HTI half-space are given in the caption of Figure 9. The largest effect of the azimuth $\psi_{2}$ and angle separation $\Delta \psi$ on the resolution of medium parameters is achieved in the postcritical domain rather than in the precritical domain. Moreover, different combinations of acquisition parameters affect the resolution of different sets of parameters. Thus, $45^{\circ}<\psi_{2}<135^{\circ}$ and $\Delta \psi<45^{\circ}$ results in the best resolution of $V_{\mathrm{P} 2}$ in the postcritical domain, as this acquisition setup provides the best coverage of the isotropic plane. The best resolution of the anisotropy parameter $\epsilon^{(V)}$ is achieved when one of the survey lines is close to the symmetry axis direction $\left(\psi_{2} \approx \Delta \psi\right)$. Although the
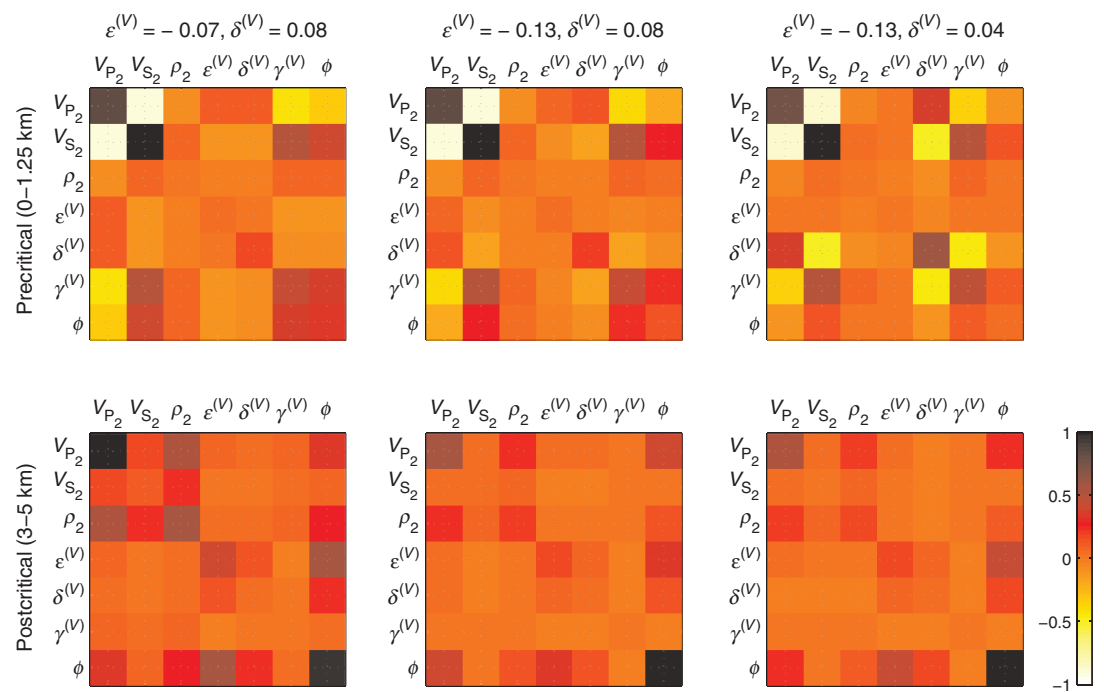

Figure 8. Resolution matrices of parameters for the HTI layer for different values of $\epsilon^{(V)}$ and $\delta^{(V)}$. Other parameters remain constant: $V_{\mathrm{P} 2}=2.8 \mathrm{~m} / \mathrm{s}, \quad V_{\mathrm{S} 2}=1.6 \mathrm{~km} / \mathrm{s}$, $\rho_{2}=2.1 \mathrm{~kg} / \mathrm{m}^{3}, \gamma^{(V)}$, and $\phi=0^{\circ}$. Acquisition parameters are $\psi_{2}=30^{\circ}, \Delta \psi=60^{\circ}$. Parameters of the upper isotropic half-space are $V_{\mathrm{P} 1}=2.0 \mathrm{~km} / \mathrm{s}, V_{\mathrm{S} 1}=1.1 \mathrm{~km} / \mathrm{s}$, and $\rho_{1}=1.8 \mathrm{~g} / \mathrm{cm}^{3}$.
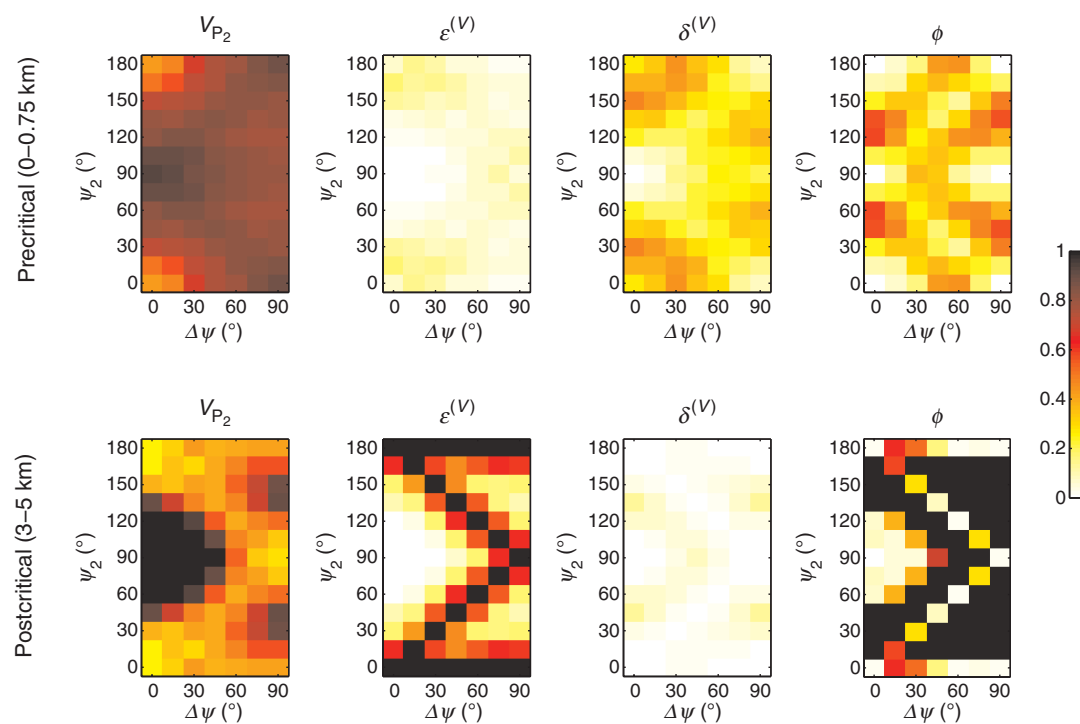

Figure 9. Dependence of some diagonal values of the resolution matrix on the acquisition parameters $\psi_{2}$ and $\Delta \psi_{2}$. The HTI medium is given by the following parameters $V_{\mathrm{P} 2}=2.8 \mathrm{~m} / \mathrm{s}, V_{\mathrm{S} 2}=1.6 \mathrm{~km} / \mathrm{s}, \rho_{2}=2.1 \mathrm{~g} / \mathrm{cm}^{3}, \epsilon^{(V)}=-0.13, \delta^{(V)}=-0.08, \gamma^{(V)}=$ -0.1 , and $\phi=0^{\circ}$. 
normalized ERC in the postcritical domain is less sensitive to the anisotropy parameter $\delta^{(V)}$, its best resolution is observed when one or more acquisition lines are close to the direction of $45^{\circ}$ from the symmetry axis $\left(\left|\psi_{2}-\Delta \psi\right| \approx 45^{\circ}\right)$. The highest resolution of the symmetry axis direction is achieved when survey lines deviate from the symmetry axis and the isotropy plane. The direction of the symmetry axis $\phi$ is better resolved with an acquisition setup with $\Delta \psi>45^{\circ}$, where the resolution matrix shows fewer blind regions than other parameters.

\section{RESOLUTION OF THE INVERSION}

To gain insights on the feasibility of applying an inversion for all or some of the parameters, we exploit the reciprocal of the condition number (Al-Dajani and Alkhalifah, 2000) $\kappa^{-1}=\sqrt{\left(\left|\lambda_{\min }\right|\right) /\left(\left|\lambda_{\max }\right|\right)}$, where $\lambda_{\min }$ and $\lambda_{\max }$ are the minimum



Figure 10. The reciprocal of the condition number $\left(\kappa^{-1}\right)$ as a function of offset, azimuth $\psi_{2}$, and separation angle $\Delta \psi$. Model parameters are given in Figure 9.


Figure 11. Two-dimensional crossplots of the objective function for different offset ranges for model parameters given in Figure 9. Squares denote true model values; circles indicate a minima in the objective function. and maximum eigenvalues of the matrix $\mathbf{M}=\mathbf{J}^{T} \mathbf{J}$, respectively. Larger $\kappa^{-1}$ values indicate better resolution in the inversion. The value of $\kappa^{-1}$ depends on the number of unknown parameters used in the inversion. To increase $\kappa^{-1}$, we have to assume some of the parameters are known.

Here, we focus on the assessment of the inversion stability for the most useful HTI parameters in practice: $V_{\mathrm{P} 2}, \epsilon^{(V)}$, and $\phi$. This implies that the matrix $\mathbf{M}=\mathbf{J}^{T} \mathbf{J}$ consists of derivatives of the normalized ERCs with respect to these three parameters only and therefore represents a $3 \times 3$ matrix. Figure 10 shows $\kappa^{-1}$ as a function of offset, azimuth $\psi_{2}$, and separation angle $\Delta \psi$. Cold colors are associated with poor parameter resolution and represent blind zones, whereas warm colors indicate good resolution. Horizontal slices, corresponding to pre-, near-, and postcritical offsets, show the best resolution of all three parameters when postcritical offsets are involved in matrix $\mathbf{M}$ evaluation. However, the influence of the azimuth angle and the separation angle in parameter resolution cannot be disregarded, and one has to be careful with the acquisition setup. The location of the survey line around the isotropic plane $\left(45^{\circ}<\psi_{2}<135^{\circ}, \Delta \psi<45^{\circ}\right)$ results in a blind zone. For optimal acquisition setup, separation angles larger than $45^{\circ}\left(\Delta \psi>45^{\circ}\right)$ are preferable. This observation is consistent with Figure 9.

To confirm the previous observations and obtain some insights into the changes in the accuracy of the parameter recovery process beyond the linear limit, we monitor changes of the shape of the objective function using postcritical reflections. For this purpose, we exploit objective functions for a single azimuth, given by

$$
\mathbf{F}=\sqrt{\sum_{n=1}^{N}\left[A^{D}\left(\mathbf{x}_{n}\right)-A\left(\mathbf{x}_{n}\right)\right]^{2}},
$$

where $A^{D}\left(\mathrm{x}_{n}\right)$ is the normalized reflection coefficient obtained from the data (Skopintseva et al., 2011) for a chosen azimuth and $A\left(\mathrm{x}_{n}\right)$ is the normalized ERC.

Figure 11 shows $2 \mathrm{D}$ crossplots of the objective function for an azimuth of $45^{\circ}$. For our calculations, we exploited the model with parameters given in Figure 9. For each plot, we vary only two parameters by $20 \%$, whereas the rest of the parameters remain constant corresponding to their true values (the minima of the objective function). The top row represents the objective functions for only precritical offsets, whereas the bottom row represents the objective functions with information from near- and postcritical offsets included. Although Figure 11 does not represent the whole set of possible 2D cross sections, the effect of postcritical offsets on the shape of the objective function is obvious. We note that the shape of the objective function is generally smooth, which implies that our linearized observations in Figures 8,9 , and 10 can be generalized. Figure 11 (top row) shows strong trade-offs between the anisotropy parameters. Specifically, the shape of the objective function along the $\phi$ and $\epsilon^{(V)} \mathrm{di}$ rections is stretched, indicating larger uncertainty compared with parameters $V_{\mathrm{P} 2}, \delta^{(V)}$, and $\gamma^{(V)}$. This observation is consistent with the resolution matrix. When the postcritical offsets are involved 
in the objective function, its shape significantly changes, indicating better resolution of $\phi$ and $\epsilon^{(V)}$.

\section{DISCUSSION}

Analysis of the reflection coefficients for an isotropic/HTI interface shows that postcritical reflections contain information about the underburden that cannot be recovered from precritical reflections. Precritical reflections have higher sensitivity to P- and S-wave velocities and anisotropy parameters $\gamma^{(V)}$ and $\delta^{(V)}$. Sensitivity analysis of postcritical reflections shows that they can potentially be inverted to recover the P-wave velocity, the symmetry axis direction, the anisotropy parameter $\epsilon^{(V)}$ with small uncertainties, and the anisotropy parameter $\delta^{(V)}$ with a larger uncertainty. Although the precritical reflections are sensitive to the direction of the symmetry axis, which is observed in some cases, the sensitivity of the postcritical reflections to this parameter is much higher. This is explained by the proportionality of the critical angle to the horizontal velocity of the underburden.

Inversion of azimuthal traveltimes (Al-Dajani and Alkhalifah, 2000) also allows the retrieval of a set of parameters, similar to inversion of postcritical reflection amplitudes. The difference between these two approaches is that inversion of azimuthal traveltimes provides information about global properties of the overburden, whereas azimuthal postcritical reflections provide knowledge about local properties of the media just above and just below the interface. A combination of these two approaches might improve resolution on the media parameters of the subsurface.

We considered the anisotropy effect on the reflection coefficient with one interface only, where the tuning effect due to interference of reflection events from neighboring interfaces is avoided. The tuning issue, however, should not be underestimated, especially for long-offset data, in which a complicated interference pattern is normally observed. This issue requires additional theoretical formulation of ERCs for a stack of layers, due to current limitations in the apparent wavefront radius definition (Ayzenberg et al., 2009; Skopintseva et al., 2011). This additional development would be an important step in generalizing the analysis of reflection amplitudes for long-offset data to multilayered media.

The sensitivity study is done for the ERC for single frequencies. However, a reflection event on a seismogram is not monochromatic. It inherits a band of frequencies admitted by the source wavelet. The presence of multifrequencies results in oscillation smoothing in the postcritical domain beyond the first amplitude peak (Skopintseva et al., 2011). However, the first peak still follows the azimuthal patterns shown in Figure 5. Therefore, it is expected that the sensitivity of a multifrequency reflection response will be similar to a singlefrequency ERC.

Even though sensitivity analysis and assessment of inversion stability are performed for a multiazimuth coverage with only three survey lines for simplicity, some of the insights gained will help in optimizing a more elaborate acquisition setup geared for parameter estimation. An azimuthal distribution of survey lines in the vicinity of the isotropic plane results in reduced stability in the inversion. An increase in stability of the inversion when postcritical reflections are included is predicted to be better than when only precritical reflections are used.

An analysis of the objective function for inversion for the subsurface parameters highlights the influence of postcritical reflections on shape of objective function minimum. Given that the ERC shows different sensitivities to parameters prior to and beyond the critical angle, joint azimuthal inversion of pre- and postcritical reflections looks most promising. Including azimuthal traveltime information in the inversion may also reduce uncertainty in parameter estimates.

\section{CONCLUSIONS}

Azimuthal analysis of the ERCs for a horizontal isotropic/HTI interface shows that the reflection amplitudes beyond the critical angle depend strongly on azimuthal variations in horizontal velocity and therefore contain additional information about the anisotropy of the underburden. The simple link given by Snell's law between the azimuthally dependent critical angle and the azimuthally dependent horizontal velocity provides an opportunity to use postcritical reflections in the recovery of anisotropy parameters. However, it is difficult in practice to retrieve the critical angle from the data. The position of the amplitude maximum beyond the critical angle can be used instead.

Sensitivity analysis of the ERCs for an isotropic/HTI interface shows that the resolution of medium parameters is highly dependent on proximity to the critical angle. Reflections prior to the critical angle are more sensitive to anisotropy parameters $\delta^{(V)}$ and $\gamma^{(V)}$, whereas reflections beyond the critical angle provide better resolution of the anisotropy parameter $\epsilon^{(V)}$ and the symmetry axis direction.

The acquisition setup also plays an important role in parameter resolution, especially in the postcritical domain. Our investigation, performed for an acquisition with minimal multiazimuthal coverage (three survey lines with constant angle of separation), shows that poor resolution is obtained when all survey lines are located close to the isotropic plane. The optimal acquisition setup, for best resolution of medium parameters, should have an azimuthal angle between survey lines of more than $45^{\circ}$ or, alternatively, a larger number of survey lines.

\section{ACKNOWLEDGMENTS}

We acknowledge Statoil for funding the Ph.D. study of L. Skopintseva and KAUST for financial support. We also thank the associate editor and the reviewers for their helpful suggestions and comments.

\section{REFERENCES}

Al-Dajani, A., and T. Alkhalifah, 2000, Reflection moveout inversion for horizontal transverse isotropy: Accuracy, limitation, and acquisition: Geophysics, 65, 222-231, doi: 10.1190/1.1444713.

Alhussain, M., 2007, Spherical wave AVO response of isotropic and anisotropic media: Laboratory experiment versus numerical simulations: M.S thesis, Curtin University of Technology.

Alkhalifah, T., and I. Tsvankin, 1995, Velocity analysis for transversely isotropic media: Geophysics, 60, 1550-1566, doi: 10.1190/1.1443888.

Avseth, P., T. Mukerji, G. Mavko, and J. A. Tyssekvam, 2001, Rock physics and AVO analysis for lithofacies and pore fluid prediction in a North Sea oil field: The Leading Edge, 20, 429-434, doi: 10.1190/1.1438968.

Ayzenberg, M., I. Tsvankin, A. Aizenberg, and B. Ursin, 2009, Effective reflection coefficients for curved interfaces in transversely isotropic media: Geophysics, 74, no. 5, WB33-WB53, doi: 10.1190/1.3197862.

Ayzenberg, M. A., A. M. Aizenberg, H. B. Helle, K. D. Klem-Musatov, J. Pajchel, and B. Ursin, 2007, Three-dimensional diffraction modeling of singly scattered acoustic wavefields based on the combination of surface integral propagators and transmission operators: Geophysics, 72, no. 5 , SM19-SM34, doi: 10.1190/1.2757616.

Červený, V., 1961, The amplitude curves of reflected harmonic waves around the critical point: Studia Geophysica et Geodaetica, 5, 319 351, doi: 10.1007/BF02585404. 
Downton, J. E. and C. Ursenbach, 2006, Linearized amplitude variation with offset (AVO) inversion with supercritical angles: Geophysics, 71, no. 5, E49-E55, doi: 10.1190/1.2227617.

Gray, F. D., G. Roberts, and K. J. Head, 2002, Recent advances in determination of fracture strike and crack density from P-wave seismic data: The Leading Edge, 21, 280-285, doi: 10.1190/1.1463778.

Grechka, V., I. Tsvankin, and J. K. Cohen, 1999, Generalized Dix equation and analytic treatment of normal-moveout velocity for anisotropic media: Geophysical Prospecting, 47, 117-148, doi: 10.1046/j.1365-2478.1999 $.00120 . x$.

Grechka, V., I. Vasconcelos, and M. Kachanov, 2006, The influence of crack shape on the effective elasticity of fractured rocks: Geophysics, 71, no. 5, D153-D160, doi: 10.1190/1.2240112.

Hall, S., and J. M. Kendall, 2003, Fracture characterization at Valhall: Application of P-wave amplitude variation with offset and azimuth AVOA analysis to a 3-D ocean-bottom data set: Geophysics, 68, 1150-1160, doi: $10.1190 / 1.1598107$.

Landrø, M., and I. Tsvankin, 2007, Seismic critical-angle reflectometry: A method to characterize azimuthal anisotropy? Geophysics, 72, no. 3, D41-D50, doi: 10.1190/1.2437145.

Lynn, H. B., D. Campagna, K. M. Simon, and W. E. Beckham, 1999, Relationship of P-wave seismic attributes, azimuthal anisotropy, and commercial gas pay in 3-D P-wave multiazimuth data, Rulison Field, Piceance Basin, Colorado: Geophysics, 64, 1293-1311, doi: 10.1190/1.1444635.

Mallick, S., K. Craft, L. Meister, and R. Chambers, 1998, Computation of principal directions of azimuthal anisotropy from $\mathrm{P}$-wave seismic data: Geophysics, 63, 692-706, doi: 10.1190/1.1444369.

Mallick, S., and L. Frazer, 1991, Reflection/transmission coefficients and azimuthal anisotropy in marine seismic studies: Geophysical Journal International, 105, 241-252, doi: 10.1111/j.1365-246X.1991.tb03459.x.
Ostrander, W. J., 1984, Plane-wave reflection coefficients for gas sands at nonnormal angles of incidence: Geophysics, 49, 1637-1648, doi: 10 $.1190 / 1.1441571$

Rüger, A., 2001, Reflection coefficients and azimuthal AVO analysis in anisotropic media: SEG.

Schoenberg, M., and J. Protazio, 1992, "Zoeppritz" rationalized, and generalized to anisotropy: Journal of Seismic Exploration, 1, 125-144.

Skopintseva, L., A. Aizenberg, M. Ayzenberg, M. Landrø, and T. Nefedkina, 2012, The effect of interface curvature on AVO inversion of near-critical and postcritical PP-reflections: Geophysics, 77, no. 5, N1-N16, doi: 10 .1190/geo2011-0298.1.

Skopintseva, L., M. Ayzenberg, M. Landrø, T. V. Nefedkina, and M. A. Aizenberg, 2011, Long-offset AVO inversion of PP reflections from plane interface using effective reflection coefficients: Geophysics, 76, no. 6, C65-C79, doi: 10.1190/geo2010-0079.1.

Tod, S., B. Taylor, R. Johnston, and T. Allen, 2007, Fracture prediction from wide-azimuth land seismic data in SE Algeria: The Leading Edge, 26 1154-1160, doi: 10.1190/1.2780786.

Tsvankin, I., 2005, Seismic signatures and analysis of reflection data in anisotropic media: Elsevier.

Tsvankin, I., J. Gaiser, V. Grechka, M. van der Baan, and L. Thomsen, 2010, Seismic anisotropy in exploration and reservoir characterization: An overview: Geophysics, 75, no. 5, 75A15-75A29, doi: 10.1190/1.3481775.

Ursenbach, C. P., A. B. Haase, and J. E. Downton, 2007, An efficient method for AVO modeling of reflected spherical waves: Journal of Seismic Exploration, 16, 79-104

$\mathrm{Xu}, \mathrm{X}$., and I. Tsvankin, 2007, A case study of azimuthal AVO analysis with anisotropic spreading correction: The Leading Edge, 26, 1552-1561, doi: 10.1190/1.2821942. 